\title{
Die versalzene Suppe - wer löffelt sie aus?
}

\author{
Tania Birner • Georg Houben
}

Online veröffentlicht: 30.1 .2015

(C) Springer-Verlag Berlin Heidelberg 2015

Wir freuen uns mit dem vorliegenden Themenheft fünf Fachbeiträge zu dem Thema „Grundwasser an der Küste und auf Inseln“ präsentieren zu können. Küstenzonen zählen zu den am dichtesten besiedelten und wirtschaftlich aktivsten Bereichen der Erde. Der daraus resultierende hohe Wasserverbrauch wird häufig aus dem Grundwasser gedeckt. Störungen des empfindlichen Gleichgewichtes zwischen Salz- und Süßwasser im Untergrund, z. B. durch Übernutzung, können zum Eindringen von Salzwasser in die Grundwasserleiter führen, wodurch diese für Jahrzehnte bis Jahrhunderte für die Nutzung verloren sind. Auf Inseln, und davon gibt es weltweit über $130.000\left(>1 \mathrm{~km}^{2}\right)$ mit ca. 500 Mio. Bewohnern, ist diese Problematik besonders kritisch, da hier eine Ersatzversorgung meist nicht möglich ist. Der oft schon strapazierte Begriff der nachhaltigen Nutzung spielt für solche Grundwasserleiter also eine besondere Rolle.

Der Nordseeraum ist wissenschaftshistorisch für die Erforschung des empfindlichen Gleichgewichtes von Salzund Süßwasser im Untergrund sehr bedeutend. Durch ihre Untersuchungen bei Amsterdam und auf der Insel Norderney entdeckten der Niederländer Baydon-Ghyben und der Deutsche Herzberg Anfang des 20. Jahrhunderts die fun- damentale Grundgleichung des hydrostatischen Gleichgewichtes zwischen Salz- und Süßwasser. Auch die erste SWIM-Tagung (Saltwater Intrusion Meeting) 1968 wurde von deutschen, dänischen und niederländischen Fachleuten initiiert. Im Juni 2014 fand die Tagung nun schon zum 23. Mal statt, diesmal in Husum, mit Besuchern aus aller Welt. Dies zeigt - wie dieses Heft - das gleichbleibend hohe Interesse an der Thematik.

Die Beiträge in diesem Heft reihen sich also in die umfangreiche internationale Literatur $\mathrm{zu}$ dem Themenkomplex ein. Geographisch spannen sie den Bogen von der deutschen Nordseeküste bis hin zu tropischen Inseln. Inhaltlich reicht das Spektrum von Betrachtungen der historischen Wasserversorgung im Marschland über Kartierungen der Küstenversalzung, Untersuchungen zu Auswirkungen anthropogener Einflüsse auf Küstengrundwasserleiter und Modellierungen von Szenarien des Meerwasserspiegelanstieges bis hin zu einer globalen Übersicht submariner Grundwasseraustritte. Wir wünschen viel Spaß beim Lesen!

Tania Birner (geb. Röper) und Georg Houben Berlin und Hannover

Dr. T. Birner $(\bowtie)$

Landesamt für Umwelt, Gesundheit und Verbraucherschutz Brandenburg (LUGV) Abteilung Ökologie, Naturschutz und Wasser, Seeburger Chaussee 2, 14476 Potsdam, Deutschland

E-Mail: Tania.Birner@LUGV.Brandenburg.de

Dr. G. Houben

Bundesanstalt für Geowissenschaften und Rohstoffe (BGR),

Arbeitsbereich „Grundwasserbeschaffenheit- und schutz“,

Stilleweg 2, 30655 Hannover, Deutschland

E-Mail: Georg.Houben@bgr.de 